\title{
CONTROLLABILITY OF PROCESSES WITH LARGE GAINS
}

\author{
Sigurd Skogestad ${ }^{*, 1}$ Antonio Araújo* \\ * Department of Chemical Engineering \\ Norwegian University of Science and Technology \\ N-7491 Trondheim, Norway
}

\begin{abstract}
There is some disagreement in the literature on whether large plant gains are a problem or not when it comes to input-output controllability. In this paper, the effect of input errors is studied and controllability requirements are derived. The input disturbances can be attenuated by the use of high gain feedback at frequencies where the gain is large, but this may not be possible. The nonlinear effect of limited input resolution causes limit cycle behavior similar to that found with relay feedback. The magnitude of these limit cycles depends on the high-frequency process gain, but is independent of the controller tuning and it can be reduced by pulse modulating the input signal, but this may cause excessive input movement. Thus, large gains at frequencies corresponding to the closedloop bandwidth may cause control problems, but large steady-state gains are not by themselves a problem.
\end{abstract}

Keywords: High gain, input disturbance, valve resolution, quantizer, limit cycle, controllability, PI-controller.

\section{INTRODUCTION}

The main goals of feedback control systems are to stabilize the plant and suppress the effect of unmeasured disturbances on the output. Having this in mind, a vital question arises: Is the process input-output controllable? There are many factors that need to be considered, and one of them is the magnitude of the process gain. The gain depends on the frequency and, for multivariable plants, also on the input direction. To quantify this, the singular values $\sigma_{i}(G(j \omega))$ of the process transfer function $G(s)$ are considered. Of particular interest are the maximum and minimum singular values, denoted $\bar{\sigma}(G)$ and $\underline{\sigma}(G)$, respectively. In this paper, for simplicity, SISO systems where $\bar{\sigma}(G(j \omega))=\underline{\sigma}(G(j \omega))=|G(j \omega)|$ are mainly considered.

\footnotetext{
1 Corresponding author: skoge@chemeng.ntnu.no.
}

It is well accepted that small process gains may cause problems, for example, with input saturation. For example, Morari (1983) states that, with unitary scaling of the inputs and desired output changes of magnitude one in terms of the 2-norm, the requirement for avoiding input saturation is $\underline{\sigma}(G) \geq 1$, that is, a minimum gain of one is required to have acceptable control.

It is less clear whether large process gains pose a problem. Skogestad and Postlethwaite (1996) consider the condition number, defined as $\gamma(G)=\bar{\sigma}(G) / \underline{\sigma}(G)$ and make the following conclusion: A large condition number may be caused by a small value of $\underline{\sigma}(G)$, which is generally undesirable. On the other hand, a large value of $\bar{\sigma}(G)$ is not necessarily a problem.

On the other hand, intuitively, a large process gain may be troublesome, because the output becomes very sensitive to the input changes. McAvoy and Braatz (2003) argue along these lines and claim that for control purposes the magnitude of steady-state process 
gain $(\bar{\sigma}(G))$ should not exceed about 50 . If this is correct then it would have important implications on the design of many processes.

The objective of this work is to study this in more detail. It is clear that the rule of McAvoy and Braatz (2003) is reasonable if feedforward control is considered because there will always be some error when implementing the input and without feedback this cannot be corrected for. However, in terms of feedback control, the rule cannot be generally true because for some classes of processes, it is well known that large process gains are not a problem. Consider, for example, feedback control of liquid level (output) using effluent flow (input). The steady-state gain is infinite due to an integrating transfer function, but it is easily controllable.

The effect of large system gains in the presence of input errors to the process will be studied in this work. Two main types of input errors are discussed: input (load) disturbance and input inaccuracy caused by limited input (valve) resolution. Most of the results are derived for first-order plus delay processes, otherwise, when appropriated, more general derivations are presented.

\section{INPUT LOAD DISTURBANCE}

It is well known that "large disturbances" (i.e. processes for which $\left|G_{d}\right| d$ is large) cause control problems. First, it causes problems for feedforward control where the disturbance is measured. This follows because to compensate for the disturbance it is needed to be very precise with the input change, and this is very difficult in practice due to model error (e.g. see eq. (5.70) in Skogestad and Postlethwaite (1996)). Thus, large disturbances motivates the need for feedback control, which is considered in this paper.

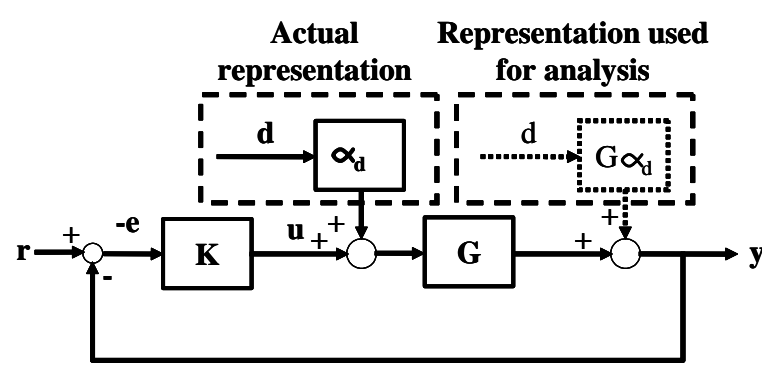

Fig. 1. Block diagram of a feedback control system with disturbance to the input to the plant.

With feedback control "large disturbances" are not necessarily a problem, but they pose limitations on the minimum bandwidth. Consider a single disturbance $d$ and assume that the reference is constant, i.e. $r=0$. Without control the steady-state sinusoidal response from $d$ to the control error is $e(\omega)=G_{d}(j \omega) d(\omega)$, where phasor notation is used. Assume that the worstcase disturbance at any frequency is $d(t)=d_{0} \sin \omega t$, i.e. $|d(\omega)|=d_{0}$, and the control objective is that the controller error is less than $e_{\max }$ at any each frequency, i.e., $|e(\omega)|<e_{\max }$. From this, one can immediately draw the conclusion that no control is needed if $\left|G_{d}(j \omega) d_{0}\right|<e_{\max }$ at all frequencies (in which case the plant is said to be "self-regulating"). If $\left|G_{d}(j \omega)\right| d_{0}>e_{\max }$ at some frequency, then control is needed (feedforward or feedback). In the following, feedback control is considered, in which case $e(s)=$ $S(s) G_{d}(s) d(s)$, where $S=(I+G K)^{-1}$ is the sensitivity function. With $|d(\omega)|=d_{0}$, the requirement $|e(\omega)|<e_{\max }$ then becomes

$$
|S(j \omega)| \cdot\left|G_{d}(j \omega)\right| d_{0}<e_{\max } \quad \forall \omega
$$

A plant with a small $\left|G_{d}\right|$ is preferable since the need for feedback control is then less, or alternatively, given a feedback controller (and thus given $S$ ), the effect of disturbances on the output is small.

$|S|$ is small at low frequencies, so in general it does not matter if $\left|G_{d}\right|$ is large at steady state. However, $|S|$ increases with frequency and crosses 1 at the bandwidth frequency $\omega_{B}$. At this frequency, which is often the worst, $\left|S\left(j \omega_{B}\right)\right|=1$ and must from (1) require

$$
\left|G_{d}\left(j \omega_{B}\right)\right|<y_{\max } / d_{0}
$$

Thus, an upper bound on the allowed disturbance gain at the frequency $\omega_{B}$ have been derived. In most cases $\left|G_{d}\right|$ becomes smaller at high frequency, so the bound is easier to satisfy if $\omega_{B}$ is increased. However, for stability reasons the value of $\omega_{B}$ is limited, and $\omega_{B}<\omega_{\mathrm{B}, \max } \approx 1 / \theta$, approximately (Skogestad and Postlethwaite, 1996), where $\theta$ denotes the "effective delay" around the feedback loop. The bound (2) then becomes

$$
\left|G_{d}\left(j \omega_{\mathrm{B}, \max }\right)\right|=\left|G_{d}(j 1 / \theta)\right|<y_{\max } / d_{0}
$$

This bound is independent of the controller, and thus provides a fundamental controllability requirement.

However, the purpose of this paper is not to consider plants for which $\left|G_{d}\right|$ is large, but rather plants for which $|G|$ is large. In practice, these are related because all plants have disturbances at the input to the plant. To this effect, consider input (load) disturbances with $G_{d}(s)=G(s) \alpha_{d}$ where $\alpha_{d}$ is a constant gain. (3) then gives the following limit on the allowed plant gain at frequency $\omega_{B}$

$$
\left|G\left(j \omega_{\mathrm{B}, \max }\right)\right|=|G(j 1 / \theta)|<1 / \alpha_{d} \cdot y_{\max } / d_{0}
$$

Input disturbances are very common and have many sources. For example, in many cases the input is a valve which receives its power from a hydraulic system (e.g. the brakes of a car) or from pressured air (many process control applications). A change (disturbance) in the power system will then cause an input disturbance. The value of $\alpha_{d}$ will vary depending on the application. If it is assumed that the system has been scaled such that the largest expected input is of magnitude 1 , then it seems reasonable that $\alpha_{d}$ is at least 0.01 , and that a typical value is 0.1 or larger. 
As an example consider the following plant $G(s)=$ $k e^{-\theta s} /(\tau s+1) ; G_{d}(s)=\alpha_{d} G(s)$ where $k=$ $|G(0)|$ is the steady-state gain of the plant. The highfrequency asymptote is $\mid G(j \omega \mid) \approx k / \tau \omega=k^{\prime} / \omega$, where $k^{\prime}=k / \tau$ is the initial slope of the step response. (4) gives the controllability requirement

$$
k / \tau=k^{\prime}<1 / \alpha_{d} \cdot 1 / \theta \cdot y_{\max } / d_{0}
$$

Thus, there exist an upper bound on the allowed value of $k^{\prime}$.

Comment. (5) seems to indicate that a plant with a large steady-state gain $k$ is fundamentally difficult to control. However, this is usually not true, because a large value of $k$ is usually accompanied by a large time constant $\tau$. For example, for an integrating process $G(s)=k^{\prime} e^{-\theta s} / s$. Thus, there is an infinite steadystate gain and also an infinite time constant.

\section{LIMITED INPUT RESOLUTION}

The implications of limited input resolution is studied here. The main reason for this is that McAvoy and Braatz (2003), based on a case study, claims that this imposes limitations on the allowed steady-state process gain.

\subsection{Controllability requirement for sinusoid responses}

Consider a simple SISO example where the plant is given by

$$
G(s)=100 /\left[(10 s+1)(s+1)^{2}\right]
$$

and the controller is

$$
K(s)=K_{c}\left(\tau_{I} s+1\right) / \tau_{I} s,
$$

which contains a dominant time constant $\tau_{I}=10$, that cancels the pole in $G(s)$, and $K_{c}=0.04$ is selected.

The block diagram of the feedback system is depicted in Figure 2.

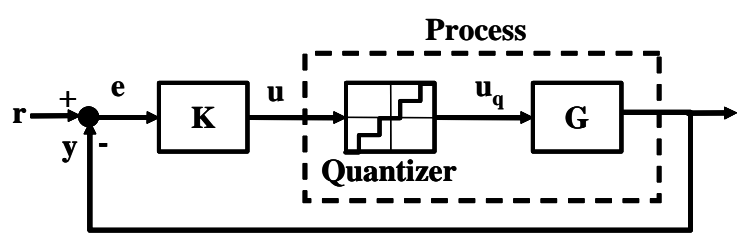

Fig. 2. Feedback control configuration for the valve inaccuracy problem.

In this Figure, $r$ is the set point, $y$ is the plant output, $u$ is the controller $(K)$ output, and $G$ is the plant. The element called quantizer has been used to simulate valve inaccuracy. The idea behind the quantizer is that it mimics the lack of resolution in the input signal by discretizing it using a constant step $q$ in order to generate the signal $u_{q}$.
The effect is thus to quantize a smooth signal into a stair-step output. The output is computed using the round-to-nearest method, which produces an output that is symmetric about zero according to (8):

$$
u_{q}=q \cdot \operatorname{round}(u / q),
$$

where $u_{q}$ is the quantizer output, $u$ is its input, $q$ the quantization step, and the round function takes its argument to the nearest integer. The limited valve resolution results in stepwise input disturbances equals to the quantization step, $q$.

For the example given by (6) and (7), $q=0.03$ is taking as the quantizer step. Figure 3 shows the response for a step change in the reference of magnitude $1\left(r_{0}=1\right)$. From this figure, the magnitude and the period of oscillations are measured to be 0.189 and $6.72 \mathrm{~s}$, respectively.

Limit cycles are inevitable if there is integral action in the controller. This follows because on average the input must equal the steady-state value $u_{s s}=$ $y_{s s} / G(0)=r / G(0)$, and if this does not happen to exactly correspond to one of the quantizer level, the quantized input $u_{q}$ will cycle between the two neighboring quantizer levels, $q_{1}$ and $q_{2}$. Let $f$ and $1-$ $f$ denote the fraction of time spend at each level. Then, at steady state $u_{s s}=f q_{1}+(1-f) q_{2}$ and from this $f$ can be found. It is noted that the closer $u_{s s}$ is to one of these values, the longer the time $u_{q}$ must remain on it. In the example, $u_{s s}=y_{s s} / G(0)=1 / 100=0.01$, which is closer to $q_{1}=0$ than $q_{2}=0.03$. The fraction of time $u_{q}$ remains on $q_{1}=0$ is $f=1-$ $0.01 / 0.3=0.67$. As expected, this agrees with the simulation.
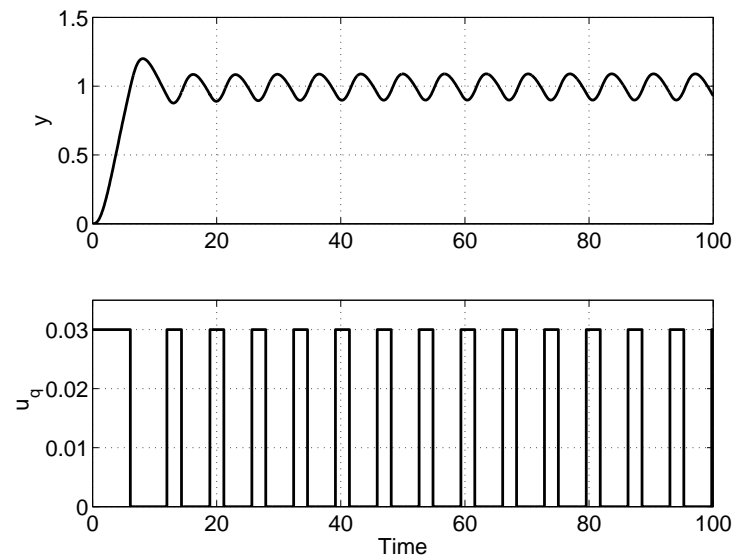

Fig. 3. Simulation results for the system given by (6) and (7) for $r_{0}=1$.

Moreover, when the limit cycle is established the quantizer can be regarded exactly as a relay without hysteresis and thus can be treated as such. The amplitude of the oscillations can then be found analytically by considering the harmonic linearization or describing function of the nonlinearity in the loop showed in Figure 2. 
For a relay without hysteresis, the describing function is given by (see Slotine and Li (1991)):

$$
N(a)=4 q / \pi a,
$$

where $a$ is the amplitude of the oscillations and $q$ is the relay amplitude (like the quantization step).

For the system depicted in Figure 2, the condition for oscillation is simply given by

$$
N(a) L(j \omega)=-1,
$$

where $L(j \omega)=G(j \omega) K(j \omega)$ is the open-loop transfer function.

Since $N(a) \in \mathbb{R}$ (see (9)), in (10), $\omega$ is the ultimate frequency $\left(\omega_{L, 180}\right)$ and $N(a)=K_{u}$ is the ultimate gain. This gives $K_{u}|L(j \omega)|=1$ and

$$
K_{u}=4 q / \pi a,
$$

Note that the gain at $\omega_{L, 180}$ is not the same as the gain at $\omega_{B}$. Actually, $\omega_{B}$ is where $|L| \approx 1$ for well-design control systems and the approximation $\omega_{L, 180}=\omega_{B}$ is quite accurate.

As long as $\tau_{I}$ in (7) is sufficiently large, that is, $\frac{1}{\tau_{I}}$ is much smaller than $\omega_{L, 180}, \omega_{L, 180}$ is independent on both $K_{c}$ and $\tau_{I}$. Thus, it can be written that $\angle K=$ $-\frac{\pi}{2}+\arctan \left(\omega_{L, 180} \cdot \tau_{I}\right) \approx 0$. Then, $\angle L=\angle G+\angle K$ gives $\angle L=\angle G \Rightarrow \omega_{L, 180}=\omega_{G, 180}$. But from frequency domain analysis $K_{u}=1 /\left|G\left(j \omega_{L, 180}\right)\right|$ and from (11)

$$
\left|G\left(j \omega_{L, 180}\right)\right|=\pi a / 4 q
$$

Let $a_{\max }$ denote the maximum allowed amplitude of the oscillations is $y$. Typically, $a_{\max }$ will be considerably smaller than $y_{\max }$, e.g. $a_{\max }=0.1 y_{\max }$. Then, from (12) the following controllability requirement applies

$$
\left|G\left(j \omega_{L, 180}\right)\right|<\pi a_{\max } / 4 q,
$$

It gives an upper limit on plant gain at frequency where $\angle L=-\pi$.

For the system given by (6) and (7), it can written that $\angle G=-\arctan \left(10 \omega_{L, 180}\right)-2 \arctan \left(1 \omega_{L, 180}\right)$. For large $\omega_{L, 180}, \arctan \left(10 \omega_{L, 180}\right)=\frac{\pi}{2} \Rightarrow \angle L=$ $\angle G=-\frac{\pi}{2}-2 \arctan \left(\omega_{L, 180}\right)$. By setting $\angle L=-\pi$, $\omega_{L, 180}=1$ and the period of oscillation is found to be $T=\frac{2 \pi}{\omega_{L, 180}}=6.28$. Moreover, from (12) and $\left|G\left(j \omega_{L, 180}\right)\right|=4.89, a=\frac{4}{\pi} q\left|G\left(j \omega_{L, 180}\right)\right|=0.187$. Consequently, the results found by the harmonic linearization approach match very accurately the simulation results.

It has been assumed here that the resulting oscillations are sinusoidal, but this may not be true. Two questions arise: is (9) an upper bound? Is (13) always true? The answer for those questions is yes, provided the response is a sinusoid-type one.
3.2 Controllability requirement for first-order plus time delay processes in time domain

In this section, nonsinusoid-type responses for a firstorder with delay plant controlled by a PI controller is discussed.

Another example is considered where

$$
\begin{array}{r}
G(s)=k e^{-\theta s} /(\tau s+1) \\
K(s)=K_{c}\left(\tau_{I} s+1\right) / \tau_{I} s,
\end{array}
$$

with $k=100, \theta=1, \tau=10, K_{c}=0.05$, and $\tau_{I}=8$.

The loop is set up according to Figure 2. The simulation results for $q=0.01$ and a step change of 0.23 in the reference $\left(r_{0}=0.23\right)$ are given in Figure 4 .
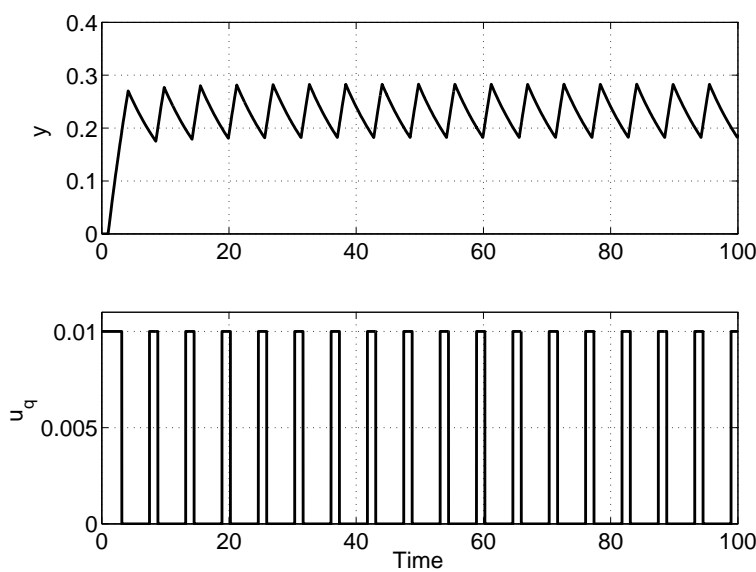

Fig. 4. Simulation results for the system given by (14) and (15)

The amplitude and period of the limit cycle of $y$ can be predicted for first-order plus delay processes as it is shown later. For this particular example they are measured to be 0.1 and $5.64 \mathrm{~s}$, respectively. It can be seen that the output of the quantizer, $u_{q}$, oscillates between 0 and 0.01 . The steady-state value is $u_{s s}=$ $0.23 / 100=0.0023$, which means that it stays $f=$ $0.23(23 \%)$ of the time (1.30s) at 0 and $77 \%(4.34 \mathrm{~s})$ at 0.01 .

Now, an exact analysis of the steady-state response, without using describing functions, is performed. The output $y$ can be represented by a sum of pulsed input responses. The resulting amplitude can be shown to be given by

$$
a=k q \frac{1-e^{-t_{1} / \tau}+e^{-T / \tau}-e^{-\left(T-t_{1}\right) / \tau}}{1-e^{-T / \tau}},
$$

where $t_{1}=\theta /(1-f), t_{2}=\theta / f, T=t_{1}+t_{2}=$ $\theta[1 /(1-f)+1 / f]$.

For the system given by (14) and (15) the amplitude and period of oscillation are given by $a=0.01$ and $T=5.64 \mathrm{~s}$, respectively which match exactly the observed results.

In general, the minimum value for $T$ and the maximum amplitude $a$ occurs when the set point change, 
$r_{0}$, is such that $f=0.5$, and $t_{1}=t_{2}=\frac{T}{2}$. In this case, $T=4 \theta$ and $a=k q\left[\left(1-e^{-2 \theta / \tau}\right)^{2} /\left(1-e^{-4 \theta / \tau}\right)\right]$.

In the case where $f=0.5$, the results also compare well with the describing function analysis based on sinusoids. For this plant, assuming $\tau_{I} \approx \tau, \angle L=$ $-\omega \theta-\pi / 2$, and $\omega_{L, 180}=\pi / 2 \theta$ and the corresponding period is $T=2 \pi / \omega_{L, 180}=4 \theta$ and $a=$ $k q \sqrt{\frac{16}{\pi^{2}} \frac{4(\theta / \tau)^{2}}{\pi^{2}+4(\theta / \tau)^{2}}}$, which for $0<\theta / \tau \leq 1$ agrees quite exactly with the previous expression for $a$.

Remark 1. Equation 16 is derived taking into account the approximation $\tau=\tau_{I}$ which applies for welltuned controllers (see Skogestad (2003)). Thus the amplitude and period of the limit cycle are independent on the controller parameters.

Again, it is required that $a<a_{\max }$. Then, the controllability requirement for first-order plus time delay processes is

$$
\begin{array}{r}
G(0)<\frac{a_{\max }}{q}\left(1-e^{-T / \tau}\right)\left[1-e^{-t_{1} / \tau}+\right. \\
\left.e^{-T / \tau}-e^{-\left(T-t_{1}\right) / \tau}\right]^{-1}
\end{array}
$$

By taking the system described by (6) and (7) and using the configuration of Figure 2 with $q=1$ (representing an on/off valve, the worst case), the simulation results for the output $y$ are depicted in Figure 5.

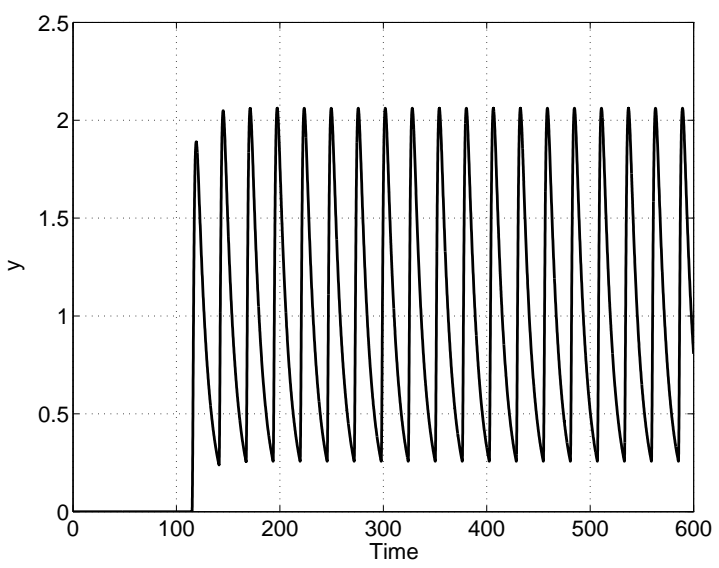

Fig. 5. Simulation results for the system given by (6) and (7) for $q=1$.

From the figure, it is clear that the oscillations are not sinusoid-type. A deeper analysis by computing the power spectrum of the limit cycle confirms this hypothesis. In Figure 6, there is a second peak of about 50 at $4 \mathrm{rad} / \mathrm{s}$ which shows the data inconsistency, i. e., the limit cycles cannot be properly fitted to a sinusoidtype curve. By using the harmonic linearization approach an amplitude of $a=6.23$ and a period of $T=6.28 \mathrm{~s}$ are found which are very different from the measured results, $a=1.82$ and $T=26.48 \mathrm{~s}$. Consequently, this approach cannot be used to assess

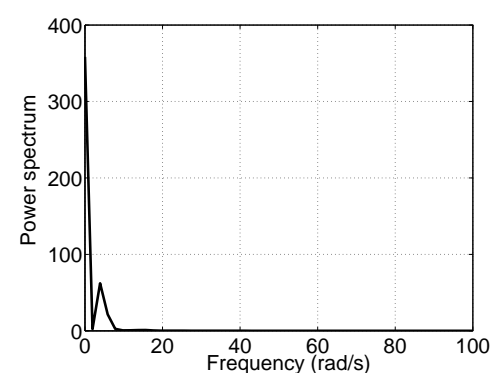

Fig. 6. Power spectrum of the limit cycle of the system described by (6) and (7) for $q=1$.

controllability. The idea now is to reduce the model to a first-order plus time delay and apply the results of this section.

The reduced model of (6) and (7) based on Skogestad (2003) paper, with some adjustments on the process gain, is given by

$$
g(s)=13 e^{-1.5 s} /(10.5 s+1)
$$

The amplitude and the period of oscillation are calculated to be $a=1.82$ and $T=21.13$, respectively, which give good accurate approximations to the simulated results.

\subsection{How to avoid oscillations}

The oscillations in the output of the system showed in Figure 2 can be avoided by the following ways:

a. Change the valve so that the resolution is enhanced (small quantization step);

b. Redesign the process in order to change the values of $\mathrm{k}, \tau$, and $\theta$;

c. Take away the integral action leaving solely a Pcontroller;

d. Introduce forced cycles at the input with a higher frequency than those generated "naturally". For example, one may use high-frequency pulse modulation or add high-frequency sinusoids.

The use of a P-controller can eliminate oscillations as long as steady-state offset can be afforded. In order to make the offset as small as possible, bounds on the controller gain, $K_{c}$, are found to be (again, for the sake of compactness, the derivation of those bounds are not to be shown here):

$$
\frac{n_{\max } q}{r_{0}-n_{\max } q G(0)} \leq K_{c}<\frac{\left(n_{\max }+0.5\right) q}{r_{0}-n_{\max } q G(0)}
$$

where

$$
n_{\text {max }}=\left\lfloor r_{0} / q G(0)\right\rfloor .
$$

An attractive alternative, at least from a theoretical point of view, is to introduce high-frequency cycling at the input. The problem is that the fast cycling may be difficult to handle in practice, for example, because the valve cannot be moved so fast or because of excessive wear. One approach is to introduce a pulse modulator 
in the controller before the quantizer. By applying this method, the response of the system given by (14) and (15) is depicted in Figure 7. As it can be seen, the amplitude is drastically reduced.

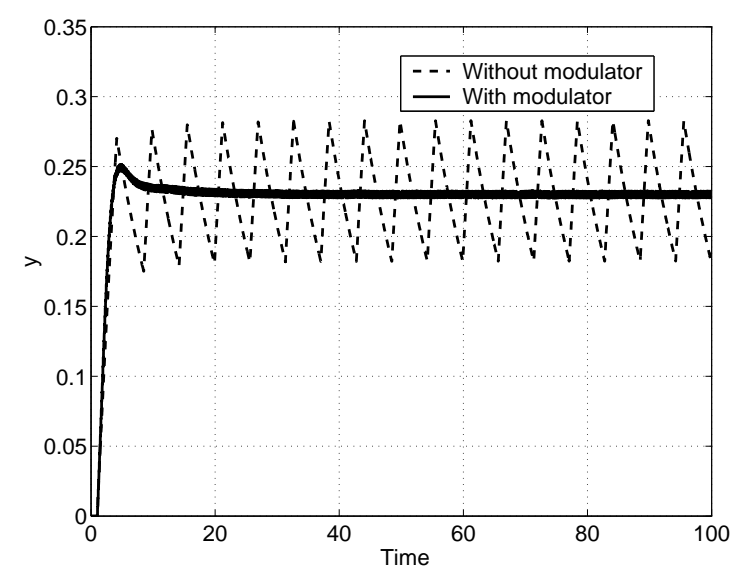

Fig. 7. Simulation results for the system given by (14) and (15) using a modulator $\left(r_{0}=0.23\right)$.

\section{DISCUSSION}

McAvoy and Braatz (2003) claim that an upper limit for $\bar{\sigma}(G)$ should be imposed. It is suggested that a reasonable limit is 50 because essentially all control systems are eventually implemented with analogue devices which typically have an accuracy on the order of $0.5 \%$. Actually this is true only at bandwidth frequency whereas no such limit exist at steady-state. Furthermore, it is also claimed that it is impossible in practice to get the fine manipulation of the control valves that is required for control because these valves would be limited to move in a very small region. Actually this is only true for feed forward systems without pulsing. There will be no problem with feedback, but some cycling must be accepted.

McAvoy and Braatz (2003) claim that the cycling can be avoided by detuning the controller, but this is not generally true, unless one is willing to remove the integral action and accept an offset. The simulation used by McAvoy and Braatz (2003) to illustrate this claim is misleading, because oscilations do start if the simulation time is increased.

An important distinction between input load disturbance and valve inaccuracy is that, in general, in the latter a high bandwidth has no effect on the controllability of the system since the controller parameter do not affect the limit cycle as showed for first-order plus delay processes.

As shown, two basic approaches to assess controllability are discussed in this paper. But in order to make use of one or the other, the resulting limit cycle has to be characterized. If the process is a first-order plus time delay the controllability requirement is directly given by (17). Otherwise, simulations must be performed in order to determine if the limit cycle is sinusoid-type, for example, by performing a spectral power analysis. If the limit cycle is proved to be sinusoid-type, (13) is used as the controllability requirement. Finally, if the limit cycle fails to be sinusoid-type it should be reduced to a first-order plus time delay and (17) be used.

\section{CONCLUSION}

Processes with large gains are a major problem when input load disturbance and valve inaccuracy problems arise. For input load disturbance, high gain implies the need of a high bandwidth which cannot always be achieved in practice.

When dealing with valve inaccuracy problems a different approach has to be used since, in general, the controller parameters do not affect the bandwidth. For sinusoid-type limit cycles, the simple approach using harmonic linearization approximation are derived to assess controllability. As for first-order plus time delay processes, on the one hand, more complicated expressions are needed to assess controllability but on the other hand, the results are exact. A general approach to deal with valve inaccuracy is proposed.

In order to avoid oscillations due to valve inaccuracy using control techniques, bounds on the controller gain for P-controllers are derived in order to keep the offset as small as possible. However, the pulse modulation approach yields much better results since the remaining oscillations are of very low amplitude.

Furthermore, pragmatic ideas on how to cope with processes with large gains are demystified in this paper.

\section{REFERENCES}

McAvoy, T. J. and R. D. Braatz (2003). Controllability of process with large singular values. Ind. Eng. Chem. Res. 42, 6155-6165.

Morari, M. (1983). Design of resilient processing plants III - a general framework for the assessment of dynamic resilience. Chemical Engineering Science 38, 1881-1891.

Skogestad, S. (2003). Simple analytic rules for model reduction and PID controller tuning. Journal of Process Control 13, 291-309.

Skogestad, S. and I. Postlethwaite (1996). Multivariable Feedback Control: Analysis and design. John Wiley \& Sons. Chichester, UK.

Slotine, J. E. and W. Li (1991). Applied Nonlinear Control. Prentice-Hall International Editions. New Jersey, USA. 